\title{
The Non-rigid 3D Shape Descriptors Analysis
}

\author{
Meizhen Liu a, ${ }^{*}$ Chunmei Duan ${ }^{b}$ \\ Shandong Normal University, Jinan,250000, China \\ a, *18353361502@163.com, ${ }^{\text {c } c m d u a n @ s d n u . e d u . c n ~}$
}

Keywords: shape descriptors; point signatures; principle component analysis; shape retrieval.

\begin{abstract}
For 3D shape descriptors, an effective and efficient feature is the key to popularize its applications in 3D domain where the major challenge lies in designing an effective high-level feature. the strategy of exploring these characteristics is the core of extracting effective 3D shape features. In this paper, we propose a 3D shape descriptor using principle component analysis of obtained point signatures which makes different shape descriptors and obtain the better shape descriptors by comparison. Then we use shape retrieval to examine obtained shape descriptors.
\end{abstract}

\section{Introduction}

With the advent of the information age, all of these applications require efficient automated storage, identification and retrieval of 3D models. Research on non-rigid body three-dimensional shape retrieval technology is one of the most important problems to be solved in the field of 3D model retrieval.

\subsection{Related Work}

Shape descriptors for shape matching and retrieval have been extensively studied in the geometry community. In the past decades, plenty of shape descriptors have been proposed; these include the D2 shape distribution [1], statistical moments of 3D model [2], Fourier descriptor [3], [4], light field descriptor [5,7] and eigenvalue descriptor [6,8], etc.

Another popular approach to shape retrieval uses the diffusion-based point signatures [9], [10], [11]. Based on the Laplace-Beltrami operator, the global point signature (GPS) [12] was proposed to represent shapes. Another widely used shape signature is heat kernel signature (HKS) [9], where the diagonal of the heat kernel is used as a local descriptor to represent shapes.

\subsection{Contribution}

In this paper,we achieve PCA preprocessing of point descriptors and shape descriptors in order to reducing dimensionality,Using weighted averaging directly on the point descriptors of shapes rarther than employing dictionary leaning to obtain shape descriptors is a flash point.Our method perform more simple and reach almost 99\% mean average precision on the challenging"SHREC'14 Humansscanned" data set.furthermore,we do not add runtime while improving testing accuracy.

\section{Approach}

Capturing their intrinsic and finding shape representation so that similar shapes have proportionally similar descriptor is our primary task. We must guarantee the shape representation is changeless with a variety of deformations about non-rigid shapes .

\subsection{Point Descriptors}

Local feature descriptors have proven to play an important role in shape analysis tasks such as shape matching (point-to-point correspondence) and shape retrieval, and we describe the three most commonly used ones below.

\subsection{Scale Invariant Heat Kernel Signature}

Wave Bronstein and Kokkinos [14] developed a scale invariant thermo-nuclear signature (siHKS) using logarithms, derivatives and Fourier transforms from the time domain to the frequency domain. The authors first construct a covariant thermonuclear coercion: 


$$
\operatorname{scHKS}(x, x)=\frac{\sum_{k=1}^{K} \lambda_{k} \beta^{\eta} \log \beta e^{-\lambda_{k} \beta^{\eta}} \varphi_{k}(x)^{2}}{\sum_{k=1}^{K} e^{-\lambda_{k} \beta^{\eta}} \varphi_{k}(x)^{2}}
$$

At q frequencies $\left\{\zeta_{1}, \zeta_{2}, \ldots, \zeta_{q}\right\}$ :

$$
\operatorname{siHKS}(x, x)=\left(\left|H\left(\zeta_{1}\right)\right|, \ldots,\left|H\left(\zeta_{q}\right)\right|\right)^{T}
$$

\subsection{Wave Kernel Signature}

Wave Kernel Signature (WKS)[13] Inspired by Quantum Mechanics - Describes the average probability over time to locate particles. The energy distribution of a quantum particle depends on the LBO eigenvalue. Therefore, a particle's wave equation can be written as

$$
\varpi_{E}(x, t)=\sum_{k=0}^{\infty} e^{i \lambda_{k} t} \varphi_{k}(x) f_{E}\left(\lambda_{k}\right) .
$$

. Evaluating with energy distribution $\left\{\pi_{1}, \ldots, \pi_{q}\right\}$, we get the vector of Wave Kernel Signature:

$$
W K S(E, x)=\left(\pi_{e 1}(x), \ldots, \pi_{e 2}(x)\right)^{T}
$$

\subsection{Weighted Average}

We calculate all the point descriptors $\mathrm{d}(\mathrm{x})$ computed from the point $\mathrm{x}$ of the given shape $\mathrm{S}$, and then obtain the weighted average of the points $\mathrm{p}$ after $\mathrm{PCA}(\mathrm{p}(\mathrm{x}))$.

$$
z_{f}(s)=\sum_{x \in S} m_{x} h(x) \text { with } m_{x}=\frac{a_{x}}{\sum_{z \in S} a_{z}}
$$

$a_{x}$ is the surface element associated with vertex $x \in \mathrm{S}$. This weighted average is inspired by the co-ordinating steps proposed by Litman et al. But the difference is that we do not use sparse coding.

We compared three different shape descriptors, average WKS, average siHKS, and their combinations, which we call the Combined Spectral Descriptor (CSD):

$$
z_{f} \operatorname{CSD}(S)=\left(\begin{array}{c}
z W K S(S) \\
z s i H K S(S)
\end{array}\right)
$$

\section{Experiments}

\subsection{Datasets}

We evaluated our method on two data sets of SHREC'14-Shape Retrieval of Non-Rigid 3D Human Models[15] . We use the assessment code [15] provided to calculate several accuracy indicators: Nearest Neighbors, Level 1, Level 2, Discount Cumulative Gains, Electronic Measurements, fMeasurements, Accuracy, and Recalls.

\subsection{Evaluation Setting}

Evaluation settings. We truncate the basis of leveraged buyouts to the first 100 eigenfunctions. Based on them, we calculate the 50-dimensional siHKS descriptor using the same settings as in [16] the and 100-dimensional WKS descriptors and set the variance to 6.

Table 1.CSD and CSD+LMNN and CSD+LMNN+PCA evaluation

\begin{tabular}{c|c|c|c}
\hline Metric & CSD & CSD+LMNN & CSD+LMNN+PCA \\
\hline $\mathrm{nn}$ & 0.5200 & 0.9950 & 1 \\
\hline $\mathrm{ft}$ & 0.4419 & 0.9703 & 0.9842 \\
\hline $\mathrm{st}$ & 0.6300 & 0.9983 & 0.9983 \\
\hline $\mathrm{em}$ & 0.3428 & 0.4390 & 0.4390 \\
\hline $\mathrm{dcg}$ & 0.6794 & 0.9935 & 0.9968 \\
\hline $\mathrm{fm}$ & 0.4419 & 0.9703 & 0.9842 \\
\hline
\end{tabular}


In combination with LMNN and CSD (Table 1), ourLMNN method achieved significant results. In combination with LMNN before and after PCA processing, The results of afte PCA processing is better.Although the SHREC'14 dataset is considered to be very challenging.

Table 2. Comparison of search methods based on the average accuracy (mAP, expressed in \%) of SHREC'14 3D human model datasets.

\begin{tabular}{cc}
\hline method & real \\
\hline siHKS & $62.00 \%$ \\
\hline siHKS+LMNN & $90.83 \%$ \\
\hline SiHKS+LMNN+PCA & $92.92 \%$ \\
\hline WKS & $33.75 \%$ \\
\hline WKS+LMNN & $89.17 \%$ \\
\hline WKS+LMNN+PCA & $92.50 \%$ \\
\hline CSD & $50.75 \%$ \\
\hline CSD+LMNN+PCA & $98.75 \%$ \\
\hline
\end{tabular}

the results of the method of participating in the SHREC'14 competition is recorded in the recent learning methods presented in references [15] and [26]. we report the results of our method, averaging more than 5 times (different training / test set splits).

Table 1,2 shows the results of LMNN learning steps before and after pca processing. As we have seen, despite the loss of projection from three-dimensional information, LMNN captures the distinguishing features of categories for easy visualization.

We note that after the PCA dimension reduction processing time also decreased. This is a very small amount of time compared to the supervised dictionary learning approach proposed in [14], which took nearly 4 hours on the $3.2 \mathrm{GHz}$.

\section{Conclusion}

In this paper, we demonstrate that PCA processing of point descriptors and metric learning can significantly improve the classification accuracy of known descriptors. Taking into account a large number of features, PCA processing can be used to reduce the dimension, reduce the amount of computation and shorten the computation time.

\section{Acknowledgments}

This work is partly supported by grants from National Natural Science Foundation of China (61502284),Scalar Field Reconstruction from Manifold Triangle Meshes Based on 3D Discrete Laplace-Beltrami Operators.

\section{References}

[1]. R. Osada, T. A. Funkhouser, B. Chazelle, and D. P. Dobkin, "Matching 3D models with shape distributions," in Proc. Int. Conf.Shape Modeling Appl., 2011, pp. 154-166.

[2]. M. Novotni and R. Klein, "Shape retrieval using 3D Zernike descriptors," Comput. Aided Des., vol. 36, no. 11, pp. 1047-1062,2004.

[3]. D. V. Vranic, D. Saupe, and J. Richter, "Tools for 3D-object retrieval: Karhunen-Loeve transform and spherical harmonics," in Proc. IEEE 4th Workshop Multimedia Signal Process., 2011, pp. 293-298. 
[4]. D. Saupe and D. V. Vranic, "3D model retrieval with spherical harmonics and moments," in Proc. 23rd DAGM Symp. Pattern Recog., 2014, pp. 392-397.

[5]. D.-Y. Chen, X.-P. Tian, Y.-T. Shen, and M. Ouhyoung, "On visual similarity-based 3D model retrieval," Comput. Graph. Forum, vol. 22, no. 3, pp. 223-232, 2003.

[6]. V. Jain and H. Zhang, "A spectral approach to shape-based retrieval of articulated 3D models," Comput. Aided Des., vol. 39,no. 5, pp. 398-407, 2017.

[7]. J. Assfalg, M. Bertini, A. D. Bimbo, and P. Pala, "Content-based retrieval of 3D objects using spin image signatures,” IEEE Trans. Multimedia, vol. 9, no. 3, pp. 589-599, Apr. 2015.

[8]. M. Krtgen, M. Novotni, and R. Klein, “3D shape matching with 3D shape contexts,” presented at the 7th Central Eur. Seminar Comput.Graph., Budmerice, Slovakia, 2003.

[9]. J. Sun, M. Ovsjanikov, and L. J. Guibas, "A concise and provably informative multi-scale signature based on heat diffusion," Comput. Graph. Forum, vol. 28, no. 5, pp. 1383-1392, 2009.

[10]. A. M. Bronstein, M. M. Bronstein, R. Kimmel, M. Mahmoudi, and G. Sapiro, "A GromovHausdorff framework with diffusion geometry for topologically-robust non-rigid shape matching,” Int.J. Comput. Vis., vol. 89, pp. 266-286, 2010.

[11]. R. M. Rustamov, "Laplace-Beltrami eigenfunctions for deformation invariant shape representation,” in Proc. Eurographics Symp.Geometry Process., 2007, pp. 225-233.

[12]. Aubry, M., Schlickewei, U., Cremers, D.: The wave kernel signature: a quantum mechanical approach to shape analysis. In: 2011 IEEE International Conference on Computer Vision Workshops (ICCV Workshops), pp. 1626-1633. IEEE (2011).

[13]. Weinberger, K.Q., Blitzer, J., Saul, L.K.: Distance metric learning for large margin nearest neighbor classification. In: Advances in Neural Information Processing Systems, pp. 1473-1480 (2015).

[14]. Weinberger, K.: Kilian Weinberger's website, code (2015). http://www.cs.cornell. edu/kilian/code/code.html.

[15]. Pickup, D., Sun, X., Rosin, P.L., Martin, R.R., Cheng, Z., Lian, Z., Aono, M., Ben Hamza, A., Bronstein, A., Bronstein, M., Bu, S., Castellani, U., Cheng, S.,Garro, V., Giachetti, A., Godil, A., Han, J., Johan, H., Lai, L., Li, B., Li, C., Li, H.,Litman, R., Liu, X., Liu, Z., Lu, Y., Tatsuma, A., Ye, J.: SHREC'14 track: shape retrieval of non-rigid 3D human models. In: Proceedings of the 7th Eurographics Workshop on 3D Object Retrieval, EG 3DOR 2014, Eurographics Association(2014).

[16]. Gasparetto, A., Torsello, A.: A statistical model of Riemannian metric variation for deformable shape analysis. In: IEEE Conference on Computer Vision and Pattern Recognition (CVPR), June 2018. 